CWRU-P14-98

astro-ph/9802343

February 1998

\title{
GRAVITATIONAL LENSING AND DARK STRUCTURES
}

\author{
Yu-Chung N. Cheng ${ }^{1}$ and Lawrence M. Krauss ${ }^{2}$ \\ Department of Physics, \\ Case Western Reserve University, \\ 10900 Euclid Ave, Cleveland, OH 44106-7079
}

\begin{abstract}
We examine whether a cosmologically significant distribution of dark galaxy group or cluster-sized objects can have an optical depth for multiple imaging of distant background sources which is comparable to that from known galaxies while at the same time producing angular splittings of the same order of magnitude. Our purpose is to explore whether such objects could realistically account for some of the observed lenses. Modeling such systems as isothermal spheres with core radii, and assuming a Schechter-type distribution function, we find that independent of the cosmology (open, flat matter dominated, or flat cosmological constant dominated) an allowed parameter range exists which is comparable in velocity dispersion to that for known compact groups of galaxies, although the preferred core radii are somewhat smaller than that normally assumed for compact groups. Dark cluster-sized objects, on the other hand, cannot reproduce the observed lensing characteristics. If the one known Dark cluster were a good representative of such a distribution, most such objects would not produce multiple images. We also present a result for the angular splitting due to an isothermal sphere lens with non-zero core radius, extending earlier work of Hinshaw and Krauss (1987). Our results are expressed as contour plots for fixed lensing probabilities, and angular splittings.
\end{abstract}

Subject headings: dark matter — galaxies: clusters: general — galaxies: fundamental parameters - galaxies: statistics — gravitational lensing

\footnotetext{
${ }^{1}$ E-mail address: yxc16@po.cwru.edu

${ }^{2}$ Also in Dept. of Astronomy, email: krauss@theory1.phys.cwru.edu
} 


\section{INTRODUCTION}

Ever since the first multiply imaged quasar was observed, it was clear that the statistics of gravitational lensing could be utilized to pin down cosmological parameters (i.e. Turner, Ostriker, \& Gott 1984; Kundić et al. 1997). Specifically, for a given cosmological model, one can predict the optical depth due to lensing by normal galaxies (presuming one has a model of normal galaxies and their distribution), and compare that with observations. Several large scale surveys have been performed, searching for multiple imaging of distant quasars by intervening galaxies, and more than a dozen such lensing events have been observed (Kochanek 1996). However, there is one slight peculiarity. While the overall frequency of lensing events, and the rough angular splittings are reasonably consistent with model expectations, in a significant fraction of the cases, the actual lensing galaxy is not visible. Given the predicted mass of the lensing systems, it is not obvious that such a large fraction should remain unresolved.

This prompts the natural question: Could the lenses be dark objects, perhaps objects related to the distribution of dark matter in the Universe - perhaps failed galaxies? This is not a new idea, and it is one which has been beset with problems. In the first place, anything close to a closure density of compact objects generally produces an optical depth for lensing which is too large (Press \& Gunn 1973). Second, if the dark objects are failed galaxies, then their properties will generally preclude producing multiple images with the observed angular splittings (Hinshaw \& Krauss 1987).

Here we examine another possibility. Could larger systems, on the scale of groups or clusters account for an observable fraction of the known lenses? The recent observation of a cluster-sized mass distribution containing one luminous galaxy (Hattori et al. 1997; Mushotzky 1997) provides some additional a posteriori motivation for considering this hypothesis.

On first glance such a possibility seems implausible. Larger systems, with larger velocity dispersions, will produce larger angular splittings, if they produce multiple images at all. Thus, it seems unlikely that such systems might reproduce the observed lensing characteristics, which, as alluded to above, are comparable to those one would predict for the known population of galaxies. However, Hinshaw and Krauss (1987; hereafter HK) demonstrated that under certain conditions a finite core radius suppresses the mean angular splitting due to isothermal sphere mass distributions. Here, we generalize the earlier HK result, and prove that this approximation is good for all lensing impact parameters inside the critical disk for multiple image formation. We then demonstrate that for a reasonable range of velocity dispersions, core radii, and total mass, assuming a Schechter-type distribution function, such systems can produce optical depths for lensing comparable to that due to known galaxies, with comparable angular splittings.

It is well known that due to a variety of selection effects (magnification biasing, etc.) 
the actual fraction of strong lensing events in any sample can differ dramatically from the naive optical depth calculation. However, because these systematic effects should be largely independent of the nature of the lenses themselves, if they are producing comparable angular splittings, etc, we need not consider these effects in detail here. In particular, we compare the calculated optical depth for lensing by dark objects to the naive optical depth for lensing by known galaxy distributions, known to be in the range of $10^{-3}$ to $10^{-4}$. Presumably, if the other selection effects are comparable, this will then imply that such systems could produce at least some fraction of the observed events. We thus derive contour plots in the parameter space of velocity dispersion and redshift for fixed total optical depth in order to explore the suggested range of dark lenses. For this range we then explore the magnitude of the predicted mean angular splittings. We conclude with a brief summary of our results.

\section{THEORETICAL FORMALISM}

\subsection{Optical Depth for Lensing}

We start with a mass density distribution with the following form:

$$
\rho(r)=\frac{\sigma^{2}}{2 \pi G\left(r^{2}+r_{\mathrm{c}}^{2}\right)}
$$

where $\sigma$ is the velocity dispersion of this system, and $r_{\mathrm{c}}$ is the core radius. In order to derive a finite total mass for the system we must assume some cutoff radius $r_{\infty}$. The total mass is then given by:

$$
m\left(<r_{\infty}\right)=\frac{2 \sigma^{2}}{G}\left[r_{\infty}-r_{\mathrm{c}} \tan ^{-1}\left(\frac{r_{\infty}}{r_{\mathrm{c}}}\right)\right]
$$

If we assume a matter density fraction $\Omega_{\mathrm{o}}$ in the Universe, then we can calculate the

number density of objects, $n$, as this fraction of the critical density $\rho_{\text {crit }}=\frac{3 \mathrm{H}_{0}^{2}}{8 \pi G}$ divided by the mass of each system:

$$
n=\Omega_{\mathrm{o}} \frac{\rho_{\text {crit }}}{m\left(<r_{\infty}\right)}=\Omega_{\mathrm{o}} \frac{3 \mathrm{H}_{\mathrm{o}}{ }^{2}}{16 \pi \sigma^{2}\left[r_{\infty}-r_{\mathrm{c}} \tan ^{-1}\left(\frac{r_{\infty}}{r_{\mathrm{c}}}\right)\right]}
$$

Following Turner, Ostriker, \& Gott (1984) we then define a lensing probability factor

$$
F=\frac{c^{3} \pi n \alpha_{0}^{2}}{\mathrm{H}_{\mathrm{o}}{ }^{3}}=\Omega_{\mathrm{o}} \frac{3 \pi^{2} \sigma^{2}}{c \mathrm{H}_{\mathrm{o}}\left[r_{\infty}-r_{\mathrm{c}} \tan ^{-1}\left(\frac{r_{\infty}}{r_{\mathrm{c}}}\right)\right]}
$$

Here, $\alpha_{0}=4 \pi\left(\frac{\sigma}{c}\right)^{2}$, is the bend angle for an isothermal sphere with core radius $r_{\mathrm{c}}=0$.

Then, the differential optical depth is:

$$
d \tau=F\left[\frac{y_{o \ell} y_{\ell s}}{y_{o s}}\right]^{2}\left[f\left(\beta_{0}\right)\right] \frac{d z_{o \ell}}{\sqrt{\Omega_{\mathrm{o}}\left(1+z_{o \ell}\right)^{3}+\Omega_{\mathrm{R}}\left(1+z_{o \ell}\right)^{2}+\Omega_{\Lambda}}}
$$


where $y_{o i}$ is the angular size distance (Peebles 1993):

$$
y_{o i} \equiv \frac{\mathrm{H}_{\mathrm{o}} a_{0} r_{i}}{c}=\frac{1}{\sqrt{\Omega_{\mathrm{R}}}} \sinh \left(\sqrt{\Omega_{\mathrm{R}}} \int_{0}^{z_{i}} \frac{d z}{\sqrt{\Omega_{\mathrm{o}}(1+z)^{3}+\Omega_{\mathrm{R}}(1+z)^{2}+\Omega_{\Lambda}}}\right)
$$

and

$$
f\left(\beta_{0}\right) \equiv 1+5 \beta_{0}-\frac{1}{2} \beta_{0}^{2}-\frac{1}{2} \sqrt{\beta_{0}}\left(\beta_{0}+4\right)^{\frac{3}{2}}
$$

We will explain the origin of the function $f\left(\beta_{0}\right)$ and the parameter $\beta_{0}$ in the next subsection. In the above equations, $a_{0}$ is the scale factor of the Universe, $r_{i}$ is the coordinate distance, $z_{i}$ is the redshift, $y_{o \ell}$ is the distance between the observer and the lensed galaxy, $y_{\ell s}$ is the distance between the lens and the source, and $y_{o s}$ is the distance between the observer and the source. $\Omega_{\mathrm{R}}$ is the curvature term, and $\Omega_{\Lambda}$ is the cosmological constant, so that $\Omega_{\mathrm{o}}+\Omega_{\mathrm{R}}+\Omega_{\Lambda}=1$ in all cases.

In a flat universe, $y_{\ell s}$ is simply $y_{o s}-y_{o \ell}$. However, in an open universe (with $\Omega_{\Lambda}=0$ ):

$$
y_{\ell s}=y_{o s} \sqrt{1+\Omega_{\mathrm{R}} y_{o \ell}^{2}}-y_{o \ell} \sqrt{1+\Omega_{\mathrm{R}} y_{o s}^{2}}
$$

If all lensing systems had the same mass and core radii, this would then be sufficient to calculate the relevant optical depths. However, if one has a distribution of masses and core radii, it is necessary to integrate over this distribution. For simplicity, we assume that these systems are distributed, like observed galaxies and clusters, with an effective Schechter function, with a parameter $L$, which is related to the total mass of the system.

$$
\phi(L) d L=\left(\frac{L}{L^{*}}\right)^{\alpha_{1}} \exp \left(-\frac{L}{L^{*}}\right) d\left(\frac{L}{L^{*}}\right)
$$

We can then consider $\sigma$ and $r_{\mathrm{c}}$ as functions of $L$, and then integrate over this distribution when deriving optical depths. We use the conventional parameter $L$ to parametrize this distribution. However, because we are interested in dark structures, $L$ should be understood to refer to mass, rather than luminosity in this case, unlike its representation for luminous objects. Although one could easily choose to other arbitrary mass distribution functions for equation (9), it seems reasonable to use the Schechter function, since this fits the distribution

of luminous galaxies. Our chief purpose here, in any case, is to explore what general mass ranges are picked out in order to fit the data, and this rather general parametrization is as useful as any other for this purpose.

\subsection{Lensing Cross Section and Angular Splitting}

In order to determine the cross section for strong lensing events, one needs to determine the bend angle, $\alpha$, of the light trace from the source, which in the case of an isothermal sphere 
with a finite core radius, is a function of (Hinshaw \& Krauss 1987) the velocity dispersion $\sigma$, impact parameter $b$, and core radius $r_{\mathrm{c}}$. The general formula for the bend angle is:

$$
\alpha(b)=\frac{4 b}{c^{2}} \int_{b}^{\infty} d r \frac{\partial \Phi}{\partial r} \frac{1}{\sqrt{r^{2}-b^{2}}}
$$

where

$$
\Phi(\vec{r})=-\int_{v} d^{3} \overrightarrow{r^{\prime}} \frac{G \rho\left(\overrightarrow{r^{\prime}}\right)}{\left|\vec{r}-\overrightarrow{r^{\prime}}\right|}
$$

HK derived a closed form approximation for $\alpha$ using a mass distribution of the form. Here, because there will be a relation between $r_{\infty}$ and the number and mass density of dark objects, we have to refine this earlier result to allow for finite $r_{\infty}$. It is simple to show that in this case, a first order expansion in $\left(r_{\mathrm{c}}^{2}+b^{2}\right) / r_{\infty}^{2}$ leads to

$$
\alpha(b)=\alpha_{0}\left(\frac{\sqrt{b^{2}+r_{\mathrm{c}}^{2}}-r_{\mathrm{c}}}{b}-\frac{b}{\pi r_{\infty}}\right)+o\left(\frac{b^{2}+r_{\mathrm{c}}^{2}}{r_{\infty}^{2}}\right)
$$

Following HK, we define $\bar{\lambda} \equiv a_{\text {cr }} / \alpha_{0}$, where

$$
a_{\mathrm{cr}}=\frac{c \alpha_{0} y_{o \ell} y_{\ell s}}{\mathrm{H}_{\mathrm{o}}\left(1+z_{\ell}\right) y_{o s}}
$$

so that

$$
\frac{b+l}{\bar{\lambda}}=\alpha(b)
$$

where $l$ is the transverse distance of the lens center from the line of sight.

Defining

$$
\begin{aligned}
\bar{b} & \equiv\left(1+\frac{a_{\mathrm{cr}}}{\pi r_{\infty}}\right) b \\
\bar{r}_{\mathrm{c}} & \equiv\left(1+\frac{a_{\mathrm{cr}}}{\pi r_{\infty}}\right) r_{\mathrm{c}}
\end{aligned}
$$

one can show

$$
\bar{b}+l=a_{\mathrm{cr}} \frac{\sqrt{\bar{b}^{2}+{\overline{r_{\mathrm{c}}}}^{2}}-\overline{r_{\mathrm{c}}}}{\bar{b}}
$$

This equation is identical in form to that derived by HK, so we can then use their results to directly write down the cross section

$$
\sigma_{\mathrm{cs}}=\pi a_{\mathrm{cr}}^{2} f\left(\beta_{0}\right)
$$

where

$$
\beta_{0}=\frac{\bar{r}_{\mathrm{c}}}{a_{\mathrm{cr}}}=\frac{r_{\mathrm{c}}}{a_{\mathrm{cr}}}+\frac{r_{\mathrm{c}}}{\pi r_{\infty}} \leq \frac{1}{2}
$$

Also note that $f\left(\beta_{0}\right)$ is zero when $\beta_{0}$ is larger than $\frac{1}{2}$. 
The bend angle not only allows us to derive the lensing cross section, but also the observed angular splitting between lensed images. HK demonstrated that for a lens located along the line of sight to the source, this splitting is given by $2 \sqrt{1-2 \beta_{0}} \alpha_{0} \frac{y_{\ell s}}{y_{o s}}$. In an appendix we present an analytic proof that this approximation is good to $10 \%$ for all values of $l$ relevant for multiple image formation. Hence we include this factor in our analysis. Note that, depending on the value of $\beta_{0}$, this can lead to significant reductions in the mean predicted splitting, allowing systems with velocity dispersions larger than those of galaxies to produce comparable angular splittings.

\section{NUMERICAL RESULTS}

Utilizing the formalism developed in the last section, we can estimate the optical depth due to dark objects of specified velocity dispersion and core radius for several different cosmologies. We consider first the results obtained without averaging over a distribution of objects, and then explore how such averaging can impact upon the optical depths obtained. We then focus on considerations of the mean angular splitting produced by such dark lenses, and finally make some comparisons to observations.

Figure 1 displays contour plots for optical depths of $10^{-3}$ (thick lines) and $10^{-4}$ (thin lines) with no averaging over a distribution of masses, but instead taking a uniform cosmic density of objects of fixed mass, leading to net density $\Omega_{0}$. Note that there are three free parameters, $\sigma, r_{\mathrm{c}}$ and $r_{\infty}$. For a fixed ratio $\frac{r_{\infty}}{r_{\mathrm{c}}}$, related to the mass per object, the integrated optical depth out to source redshift $z_{s}$ is then simply a function of $\sigma^{2} / r_{\mathrm{c}}$, so it seems reasonable to present the results in the phase space for this ratio, normalized in units of $\frac{(\mathrm{km} / \mathrm{sec})^{2}}{h^{-1} M p c}$ vs $z_{s}$. Comparison to existing, or proposed, mass distributions can be done by plugging in physical values for $\sigma, r_{\mathrm{c}}$, or both. Finally, all the black curves correspond to $\frac{r_{\infty}}{r_{\mathrm{c}}}=10$, and all gray curves correspond to $\frac{r_{\infty}}{r_{\mathrm{c}}}=100$. The solid curve sets are for a flat universe, with $\Omega_{\mathrm{o}}=1$, the dash-dash curve sets are for a flat universe model with $\Omega_{0}=0.3$, and $\Omega_{\Lambda}=0.7$, and the dash-dot curve sets are for an open universe model with $\Omega_{\mathrm{o}}=0.3$ and zero cosmological constant.

Note first that the $\frac{r_{\infty}}{r_{\mathrm{c}}}=100$ curve is higher than the $\frac{r_{\infty}}{r_{\mathrm{c}}}=10$ curve for a fixed cosmological model. This can be understood as follows. For a fixed value of $r_{\mathrm{c}}$ and $\sigma$, increasing $\frac{r_{\infty}}{r_{\mathrm{c}}}$ increases the mass per system. However, since the overall number density of systems is determined by the requirement of some fixed $\Omega_{\mathrm{o}}$, this number density then decreases inversely with $\frac{r_{\infty}}{r_{\mathrm{c}}}$, leading to fewer lenses. Since the lensing probability for isothermal spheres (with core radius) is not determined by their total mass, but rather by their velocity dispersion (and $r_{\mathrm{c}}$ ), having fewer lenses means a smaller optical depth, even though the mass per lens increases. Next note that while it is known that for a fixed density of lenses, the optical depth increases for an open universe model compared to a flat one $\Omega_{\mathrm{o}}=1$, this effect is not seen here simply because we normalize the number density by the matter density. Since an 
open universe has a lower matter density than a flat matter dominated universe, the number density of lenses decreases in proportion, and this is reflected in the optical depth contour plots shown here. The well known fact that the optical depth for a cosmological constant dominated flat universe is larger than for a matter dominated flat universe is also reflected in our results.

Before proceeding to compare with observational data to determine if these estimates might correspond to reasonable structures, we need to allow for the fact that the dark lenses are distributed over different masses as per equation (9).

Without strong theoretical guidance in this regard, we choose, by analogy with luminous objects, $\alpha_{1}=-1$ in equation (9). We also assume $\frac{r_{\mathrm{c}}}{\sigma^{2}}$ is proportional to $L^{\alpha_{2}}$, where we choose $\alpha_{2}$ to be negative to avoid a singularity. We display our results for $\alpha_{2}=-0.5$ and -1.5 . Note that in their lensing probability analysis for luminous objects, Krauss and White (1992) suggested that $\alpha_{2}$ could be a small positive number, although not much larger than zero at best. Note also, however that if one assumes the core radius is not a function of mass, then $\alpha_{2}$ is negative. In Figs. 2 and 3 our results for the two different choices of $\alpha_{2}$ are shown. As can be seen, the curves move toward smaller values of $\frac{\left(\sigma^{*} / \mathrm{km} / \mathrm{sec}\right)^{2}}{r_{c}^{*} /\left(h^{-1} \mathrm{Mpc}\right)}$ when $\alpha_{2}$ decreases. That is, the probability is higher for a given source redshift and $\frac{\left(\sigma^{*} / k m / s e c\right)^{2}}{r_{c}^{*} /\left(h^{-1} M p c\right)}$, when $\alpha_{2}$ decreases, as one might expect, since this implies that larger core radii are less heavily weighted. We should remind our readers here that $\sigma^{*}$ and $r_{\mathrm{c}}^{*}$ are normalized to $L^{*}$ in equation (9).

We next consider the mean angular splitting induced by the lensing distribution. Again, we first consider the case when we do not integrate over a mass distribution of lensing. Recall that for a singular isothermal sphere distribution, the mean angular splitting is $\alpha_{0}$. In the case of finite core radius, the expectation value of $\frac{\Delta \theta}{\alpha_{0}}$, using our result that $\Delta \theta \approx$ $2 \sqrt{1-2 \beta_{0}} \alpha_{0} \frac{y_{\ell s}}{y_{o s}}$, is:

$$
\begin{array}{r}
<x>\equiv \frac{1}{\tau} \int d \tau x \\
<\frac{\Delta \theta}{\alpha_{0}}>=\frac{1}{\tau} \int d \tau \frac{\Delta \theta}{\alpha_{0}}
\end{array}
$$

where $\tau$ is the optical depth from equation (5). In Fig. 4, we show contour plots for the case when the expectation values of the angular splitting either 0.5 or 0.8 times that expected for the equivalent singular isothermal sphere distribution. The thicker curves represent the expectation value of $0.8 \alpha_{0}$, and the thinner curves represent the expectation values of $0.5 \alpha_{0}$. Other symbols are the same as in Fig. 1. The vertical axis is still $\frac{(\sigma / k m / s e c)^{2}}{r_{\mathrm{c}} /\left(h^{-1} M p c\right)}$ and the horizontal axis is source redshift.

Note that the $\frac{r_{\infty}}{r_{\mathrm{c}}}=10$ curve is higher than the $\frac{r_{\infty}}{r_{\mathrm{c}}}=100$ curve. This is opposite to the probability plots discussed in the earlier subsections, and reflects the simple fact that larger $r_{\mathrm{c}}$ for a fixed $\sigma$ results in a smaller angular splitting. This tension between angular splitting and optical depth is important, because it implies that to keep angular splittings 
small enough to be comparable to those observed, one cannot allow too large a value of $\sigma$ and still keep the overall optical depth comparable to that due to luminous galaxies. This tuning is an important feature which constrains this scenario.

Finally, we repeat the above analysis, including an integration over a Schechter distribution of lenses, as described above. The parameters are the same as discussed above. In Fig. 5, we chooose $\alpha_{2}=-0.5$, and the thick and thin curves represent when the expectation values of the angular splitting are respectively 0.9 and 0.7 of the zero core radius case. In Fig. 6, we choose $\alpha_{2}=-1.5$ and the thick and thin curves represent when the expectation values of the angular splitting are 0.85 and 0.95 of the zero core radius case. Note that when $\alpha_{2}$ decreases, the expectation values for the angular splitting approach the isothermal sphere result, as one might expect, since for these values, the core radius is becoming less significant. Thus, if $\alpha_{2}$ is less negative than we have assumed here, the expectation value of the angular splitting could even be smaller in comparison to the equivalent isothermal sphere distribution than we have found.

\section{COMPARISON WITH OBSERVATIONS AND CONCLUSIONS}

Over a dozen multiply imaged quasars have now been observed in various optical and radio surveys. While these have angular splittings characteristic of those one might expect to be induced by galaxies (i.e. $O\left(2^{\prime \prime}-3^{\prime \prime}\right)$ ), several have no observable lensing systems, even when one might expect the lensing galaxy to be resolvable. When one calculates the optical depth for lensing by known galaxy distributions, ignoring magnification biasing, one typically finds $10^{-4} \leq \tau \leq 10^{-3}$. Thus, one might expect that a predicted optical depth of as low as $10^{-5}$ for some other distribution would produce at least one or two lenses in the existing surveys. In fitting the optical depth, however, one must confront the tension between optical depth and angular splitting discussed above (also compare Fig. 1 and Fig. 4). Systems with velocity dispersion greater than about $450 \mathrm{~km} / \mathrm{sec}$ would require the expectation value of the angular splitting to be less than half that predicted for an equivalent singular isothermal sphere. This, in turn, requires $r_{\mathrm{c}}$ to be larger relative to $\sigma$, which in turn, however, suppresses the optical depth. Nevertheless, in Table 1 we display several sample values of core radii and velocity dispersions which would be expected to produce a fraction of the observed lenses with angular splittings comparable to those observed. In this table we have fixed $\frac{r_{\infty}}{r_{\mathrm{c}}}=10$, set the source redshift to 3, and fixed the parameters so that the mean predicted angular splitting is 2.5 arcsec. Note that the parameter range is comparable to compact groups of galaxies, which have compatible $\frac{(\sigma / \mathrm{km} / \mathrm{sec})^{2}}{r_{\mathrm{c}} /\left(h^{-1} M \mathrm{cc}\right)}$. For example, one study suggests $\sigma$ is around 331 $\mathrm{km} / \mathrm{sec}$, and $r_{\mathrm{c}}$ is around $15 \mathrm{~h}^{-1} \mathrm{kpc}$ (Mendes de Oliveira \& Giraud 1994; Pildis, Bregman, $\&$ Evrard 1995). These numbers give $\frac{(\sigma / \mathrm{km} / \mathrm{sec})^{2}}{r_{\mathrm{c}} /\left(h^{-1} \mathrm{Mpc}\right)} \approx 7.3 * 10^{6}$, as compared to the preferred values of $\frac{(\sigma / \mathrm{km} / \mathrm{sec})^{2}}{r_{\mathrm{c}} /\left(h^{-1} \mathrm{Mpc}\right)}$ between $10^{7}$ and $10^{9}$ to result in an optical depth comparable to that of the known galaxy distribution. Also note that if we were to integrate over a distribution 
of such objects, our earlier arguments suggest that comparable optical depths and splittings could be obtained even if the mean value of $r_{c}$ were somewhat larger than given in the table.

However cluster-sized objects do not seem to be viable candidates for dark lenses because of the larger predicted splittings when multiple image formation does occur, and more importantly because the predicted optical depth is too small. (The $r_{\mathrm{c}}$ of a regular cluster is large, but the velocity dispersion is only about 2.5 times larger than that of compact groups of galaxies (Zabludoff et al. 1993). ) This conclusion is reinforced by the recent observation of an actual dark cluster by Hattori et. al. (1997) in the lensing system, MG2016+112. Based on the inferred mass of the object using X-Ray estimates of the potential, its size, and the size of the core radius of this system, we find that $\beta_{0}>1 / 2$, implying that the cluster is not responsible for the observed multiple images in this system. This is again supported by the small angular splitting of 3.4 arcsec between the images, which is characteristic of the one observed galaxy in this system.

Thus, perhaps paradoxically, the only known example of a large-scale dark object suggests that such cluster-scale objects, even if they have a significant mass density in the universe, are probably largely irrelevant for the statistics of multiply imaging distant quasars. Rather, dark objects on intermediate scales, between galaxy and cluster scales, are more likely possible candidates for dark lenses. A very recent paper which has shown a very high rate of galaxy lensing in radio surveys may be significant in this regard (Jackson et al. 1998). We also note both that a recent study of the luminosity function of the compact groups of galaxies also lends support to our choice of Schechter $\alpha_{1}=-1$ (Zepf, de Carvalho, \& Ribeiro 1997), and that the currently favored cosmological model involving a flat universe with cosmological constant (i.e. Krauss 1998) produces the largest optical depths at high redshift for

given $\frac{(\sigma / k m / s e c)^{2}}{r_{\mathrm{c}} /\left(h^{-1} M p c\right)}$, as one would expect, based on the increase in the optical depth for lensing by galaxies in this cosmology.

Clearly, in order to know whether dark clusters are important for lensing in the actual universe, larger surveys will be required, in order to reliably determine how many lensing events might not be associated with galaxies. If such events continue to be observed, our analysis suggests that the distribution of angular splittings will be an important observable which might constrain possible models.

\section{ACKNOWLEDGMENTS}

This research work has been partially supported by the Industrial Physics Group in the Physics Department at Case Western Reserve University, and by a grant from the DOE. 


\section{A. APPENDIX}

We prove here the angular splitting, $\Delta \theta$, is a monotonically decreased function of $L \equiv \frac{l}{a_{\mathrm{cr}}}$ (not to be confused with the quantity $L$ in the Schechter distribution function used in the text), where $l$ is the distance from the line of sight of the lens. Then, using this fact, we can show that $\Delta \theta$ is well approximated by $2 \sqrt{1-2 \beta} \alpha_{0} \frac{y_{\ell s}}{y_{o s}}$, the angular splitting derived by Hinshaw and Krauss for lenses along the line of sight. We start by redefining the parameters of equation (14) in Hinshaw \& Krauss (1987):

$$
x \equiv \frac{b}{a_{\mathrm{cr}}} \quad \beta \equiv \frac{r_{\mathrm{c}}}{a_{\mathrm{cr}}} \quad L \equiv \frac{l}{a_{\mathrm{cr}}}
$$

We then find

$$
x^{3}+2 L x^{2}+\left(L^{2}+2 \beta-1\right) x+2 L \beta=0
$$

(We remind the reader that $\beta$ is between 0 and $\frac{1}{2}$, and $|L| \leq 1$.) The above algebraic equation is solvable. Setting $x=y-\frac{2 L}{3}$ the solution is

$$
y=u+v, \quad-\frac{1}{2}(u+v)+i \frac{\sqrt{3}}{2}(u-v), \quad-\frac{1}{2}(u+v)-i \frac{\sqrt{3}}{2}(u-v)
$$

with

$$
\begin{gathered}
u=\sqrt[3]{-\frac{q}{2}+\sqrt{\left(\frac{q}{2}\right)^{2}+\left(\frac{p}{3}\right)^{3}}}, \quad v=\sqrt[3]{-\frac{q}{2}-\sqrt{\left(\frac{q}{2}\right)^{2}+\left(\frac{p}{3}\right)^{3}}} \\
-p \equiv 1-2 \beta+\frac{1}{3} L^{2} \geq 0 \\
\frac{q}{2} \equiv \frac{L}{3}\left(1+\beta-\frac{1}{9} L^{2}\right) \geq 0
\end{gathered}
$$

If we want to have three real solutions of $y$, which correspond to three lensing images, then $u-v$ should be a pure imaginary number. However, $u+v$ is a real number, and this gives us $u^{*}=v$, and $\sqrt{\left(\frac{q}{2}\right)^{2}+\left(\frac{p}{3}\right)^{3}}$ is a pure imaginary number. We thus define

$$
\begin{gathered}
-r^{2} \equiv\left(\frac{q}{2}\right)^{2}+\left(\frac{p}{3}\right)^{3} \leq 0 \\
r^{2}=\frac{1}{27}\left[(1-2 \beta)^{3}-L^{2}\left(2+10 \beta-\beta^{2}\right)+L^{4}\right] \geq 0
\end{gathered}
$$

and

$$
r=0 \Leftrightarrow L_{0}^{2}=\frac{l_{0}^{2}}{a_{\mathrm{cr}}^{2}}=f(\beta)
$$

so we can re-write $u$ and $v$ as following:

$$
u=-\sqrt[6]{\left(\frac{q}{2}\right)^{2}+r^{2}} \exp (-i \phi / 3), \quad v=-\sqrt[6]{\left(\frac{q}{2}\right)^{2}+r^{2}} \exp (i \phi / 3)
$$


where

$$
\tan \phi=\frac{2 r}{q} \geq 0
$$

this implies that

$$
0 \leq \phi \leq \frac{\pi}{2}
$$

Recall that $r^{2}+\left(\frac{q}{2}\right)^{2}=\left(\frac{-p}{3}\right)^{3}$, then we have

$$
u=-\sqrt{\frac{-p}{3}} \exp (-i \phi / 3), \quad v=-\sqrt{\frac{-p}{3}} \exp (i \phi / 3)
$$

we can write down the three solutions of $y$ :

$$
\begin{array}{ll}
y_{1}= & =-2 \sqrt{\frac{-p}{3}} \cos \left(\frac{\phi}{3}\right)<0 \\
y_{2}=-\frac{1}{2}(u+v)+i \frac{\sqrt{3}}{2}(u-v) & =2 \sqrt{\frac{-p}{3}} \cos \left(\frac{\pi}{3}+\frac{\phi}{3}\right) \geq 0 \\
y_{3}=-\frac{1}{2}(u+v)-i \frac{\sqrt{3}}{2}(u-v) & =2 \sqrt{\frac{-p}{3}} \cos \left(\frac{\pi}{3}-\frac{\phi}{3}\right)>y_{2}
\end{array}
$$

therefore, the angular splitting is:

$$
\frac{\Delta \theta}{\frac{y_{\ell s}}{y_{o s}}}=\alpha_{0}(\Delta(x+L))=\alpha_{0} \Delta y=\alpha_{0}\left(y_{3}-y_{1}\right)=2 \alpha_{0} \sqrt{-p} \cos \left(\frac{\pi}{6}-\frac{\phi}{3}\right)>0
$$

Based on the above equation, one can derive (after some work) that:

$$
\begin{aligned}
\frac{1}{\alpha_{0} \frac{y_{\ell s}}{y_{o s}}} \frac{d^{2}(\Delta \theta)}{d L^{2}}= & \frac{2}{3} \frac{1}{(\sqrt{-p})^{3}} \cos \left(\frac{\pi}{6}-\frac{\phi}{3}\right)\left[(1-2 \beta)-\frac{1}{3}(-p)^{2}\left(\frac{d \phi}{d L}\right)^{2}\right] \\
& +\frac{2}{3} \sin \left(\frac{\pi}{6}-\frac{\phi}{3}\right)\left[\frac{L}{3 \sqrt{-p}} \frac{d \phi}{d L}+\frac{d}{d L}\left(\sqrt{-p} \frac{d \phi}{d L}\right)\right] \leq 0
\end{aligned}
$$

Note that $\Delta \theta$ varies from $L=0$ to $L=L_{0}$ :

$$
\begin{aligned}
\Delta \theta & =2 \sqrt{1-2 \beta} \alpha_{0} \frac{y_{\ell s}}{y_{o s}}, \quad \text { when } L=0 \\
\Delta \theta & =\sqrt{3} \sqrt{1-2 \beta+\frac{1}{3} L_{0}^{2}} \alpha_{0} \frac{y_{\ell s}}{y_{o s}}, \quad \text { when } L=L_{0}
\end{aligned}
$$

Finally, the average ratio of $\Delta \theta$ to $2 \sqrt{1-2 \beta} \alpha_{0} \frac{y_{\ell s}}{y_{o s}}$ at $L=L_{0}$ is

$$
2 \int_{0}^{\frac{1}{2}} d \beta \frac{\Delta \theta}{2 \sqrt{1-2 \beta} \alpha_{0} \frac{y_{\ell s}}{y_{o s}}} \simeq 0.88
$$

Since we have shown that $\Delta \theta$ is a monotonically decreasing function of $L$, this implies that over the relevant range of $L, \Delta \theta$ does not differ by more than $10 \%$ from its value at $L=0$. 


\section{REFERENCES}

Condon, E. U., \& Odishaw, H. 1958, Handbook of Physics (McGraw-Hill Book Company, Inc)

Fukugita, M., Futamase, T., Kasai, M., \& Turner, E. L. 1992, ApJ, 393, 3

Fukugita, M., \& Turner, E. L. 1991, MNRAS, 253, 99 (FT)

Hattori, M., Ikebe, Y., Asaoka, I., Takeshima, T., Böhringer, H., Mihara, T., Schindler, S., Tsuru, T., \& Tamura, T. 1997, Nature, 388, 146

Hinshaw, G., \& Krauss, L. M. 1987, ApJ, 320, 468 (HK)

Jackson, N., Helbig, P., Browne, I. W. A., Fassnacht, C. D., Koopmans, L. V. E., Marlow, D. R., \& Wilkinson, P. N. 1998, A\&A, in press (astro-ph/9804136)

Mendes de Oliveira, C., \& Giraud, E. 1994, ApJ, 437, L103

Kochanek, C. S. 1993, ApJ, 419, 12

Kochanek, C. S. 1996, ApJ, 466, 638

Krauss, L. M., \& White, M. 1992, ApJ, 394, 385

Krauss, L. M. 1998, ApJ, 501, 461

Kundić, T., Turner, E. L., Colley, W. N., Gott, J. R., III, Rhoads, J. E., Wang, Y., Bergeron, L. E., Gloria, K. A., Long, D. C., Malhotra, S., \& Wambsganss, J. 1997, ApJ, 482, 75

Loveday, J., Peterson, B. A., Efstathiou, G., \& Maddox, S. J. 1992, ApJ, 390, 338

Mushotzky, R. 1997, Nature, 388, 126

Peebles, P. J. E. 1993, Principles of Physical Cosmology (Princeton: Princeton University Press)

Pildis, R. A., Bregman, J. N., \& Evrard, A. E. 1995, ApJ, 443, 514

Press, W. H., \& Gunn, J. E. 1973, ApJ, 185, 397

Schechter, P. L. 1976, ApJ, 203, 297

Wolfram, S. 1991, Mathematica (Addison-Wesley Publishing Company, Inc)

Turner, E. L., Ostriker, J. P., \& Gott, J. R. 1984, ApJ, 284, 1 (TOG)

Zabludoff, A. I., Geller, M. J., Huchra, J. P., \& Vogeley, M. S. 1993, AJ, 106, 1273 
Zepf, S. E., de Carvalho, R. R., \& Ribeiro, A. L. B. 1997, ApJL, 488, 11 
TABLE 1

Sample Values of Core Radit and Velocity Dispersion Relevant to Lensing

\begin{tabular}{ccccc}
\hline \hline model & $\sigma(\mathrm{km} / \mathrm{sec})$ & $r_{\mathrm{c}}\left(h^{-1} \mathrm{kpc}\right)$ & $\tau$ & $<\Delta \theta>$ \\
\hline$\Omega_{\mathrm{o}}=1, \Omega_{\Lambda}=0$ & 427 & 4.6 & $2.9 * 10^{-5}$ & $0.47 \alpha_{0}$ \\
$\Omega_{\mathrm{o}}=0.3, \Omega_{\Lambda}=0.7$ & 369 & 5.0 & $6.9 * 10^{-5}$ & $0.55 \alpha_{0}$ \\
$\Omega_{\mathrm{o}}=0.3, \Omega_{\mathrm{R}}=0.7$ & 416 & 4.4 & $3.4 * 10^{-5}$ & $0.50 \alpha_{0}$ \\
\hline
\end{tabular}

Note. - The values are obtained by assuming $\frac{r_{\infty}}{r_{\mathrm{c}}}=10$, source redshift at 3 , and $\langle\Delta \theta\rangle$ is fixed at 2 ".5. 


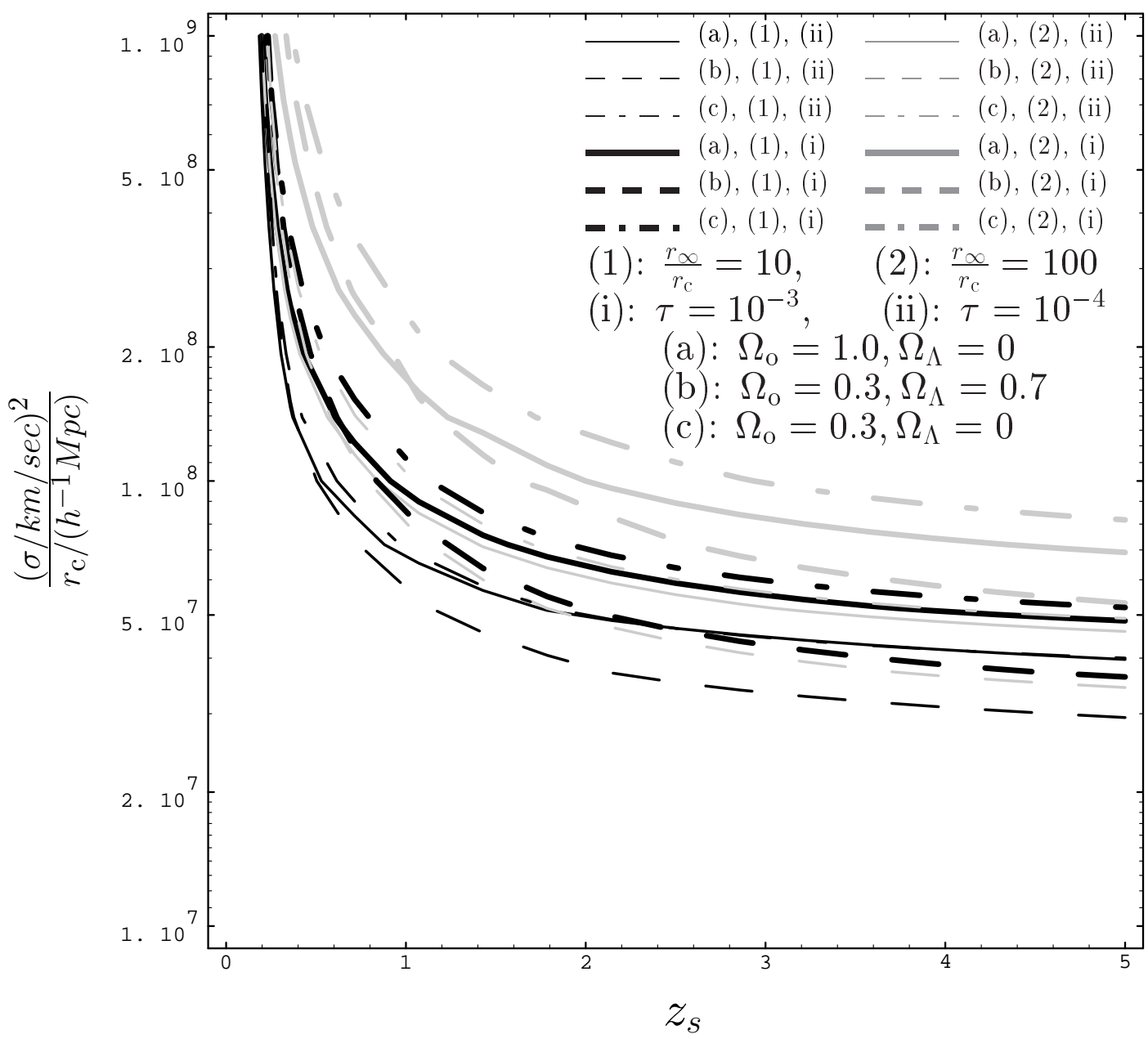

Fig. 1. - Contour plots of lensing probability for fixed lens mass. The thicker width lines represent an optical depth of $10^{-3}$, and the thinner width lines represent an optical depth of $10^{-4}$. The black curves correspond to $\frac{r_{\infty}}{r_{\mathrm{c}}}=10$, and the gray curves correspond to $\frac{r_{\infty}}{r_{\mathrm{c}}}=100$. The solid curve sets are for a flat universe model, with $\Omega_{\mathrm{o}}=1$. The dash-dash curve sets are also for a flat universe model, but with $\Omega_{\mathrm{o}}=0.3$, and $\Omega_{\Lambda}=0.7$. The dash-dot curve sets are for an open universe model, with $\Omega_{\mathrm{o}}=0.3$ and zero cosmological constant. 


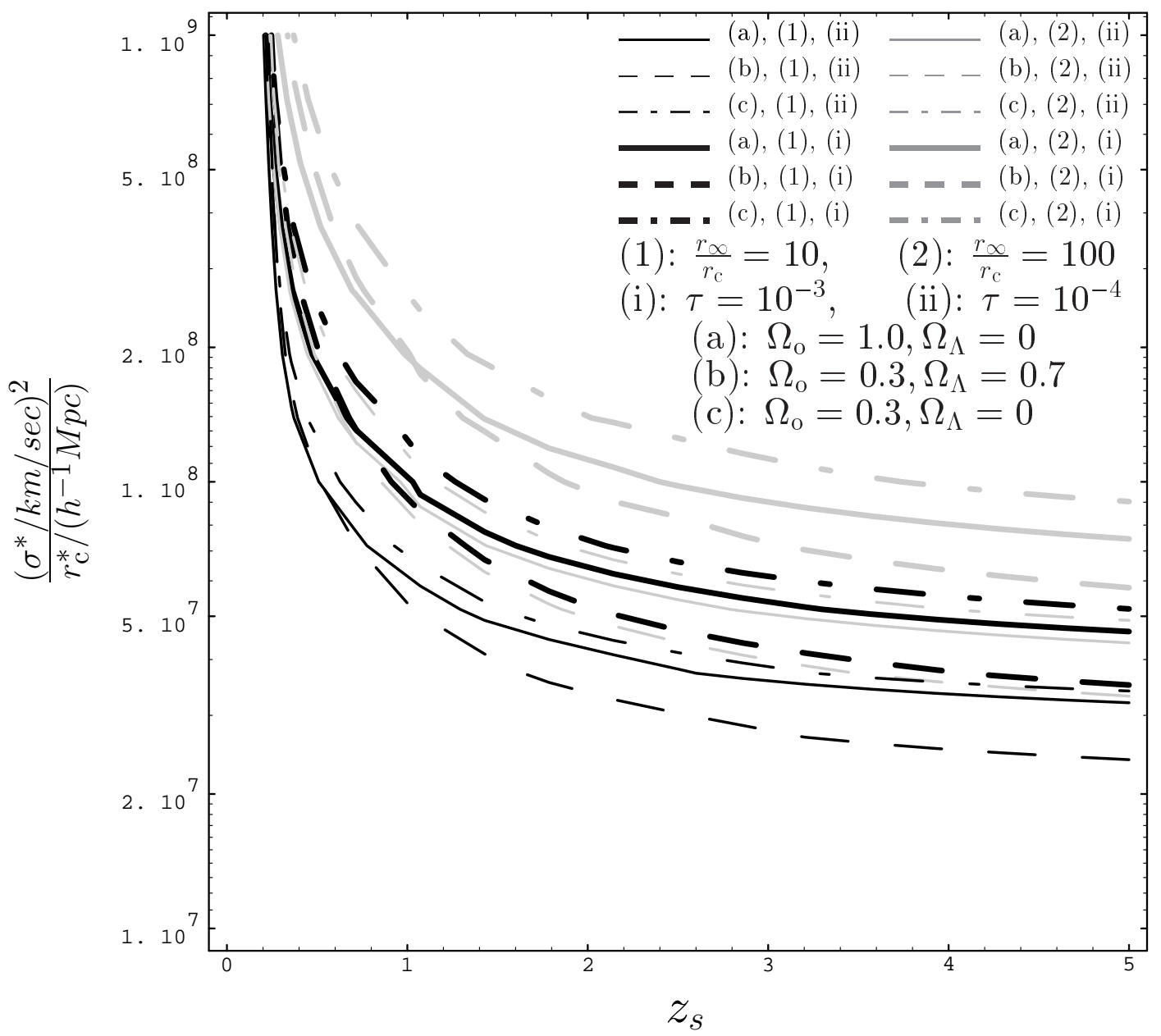

Fig. 2.- Contour plots of lensing probability as for Fig. 1, but integrating over a Schechter distribution of lenses 


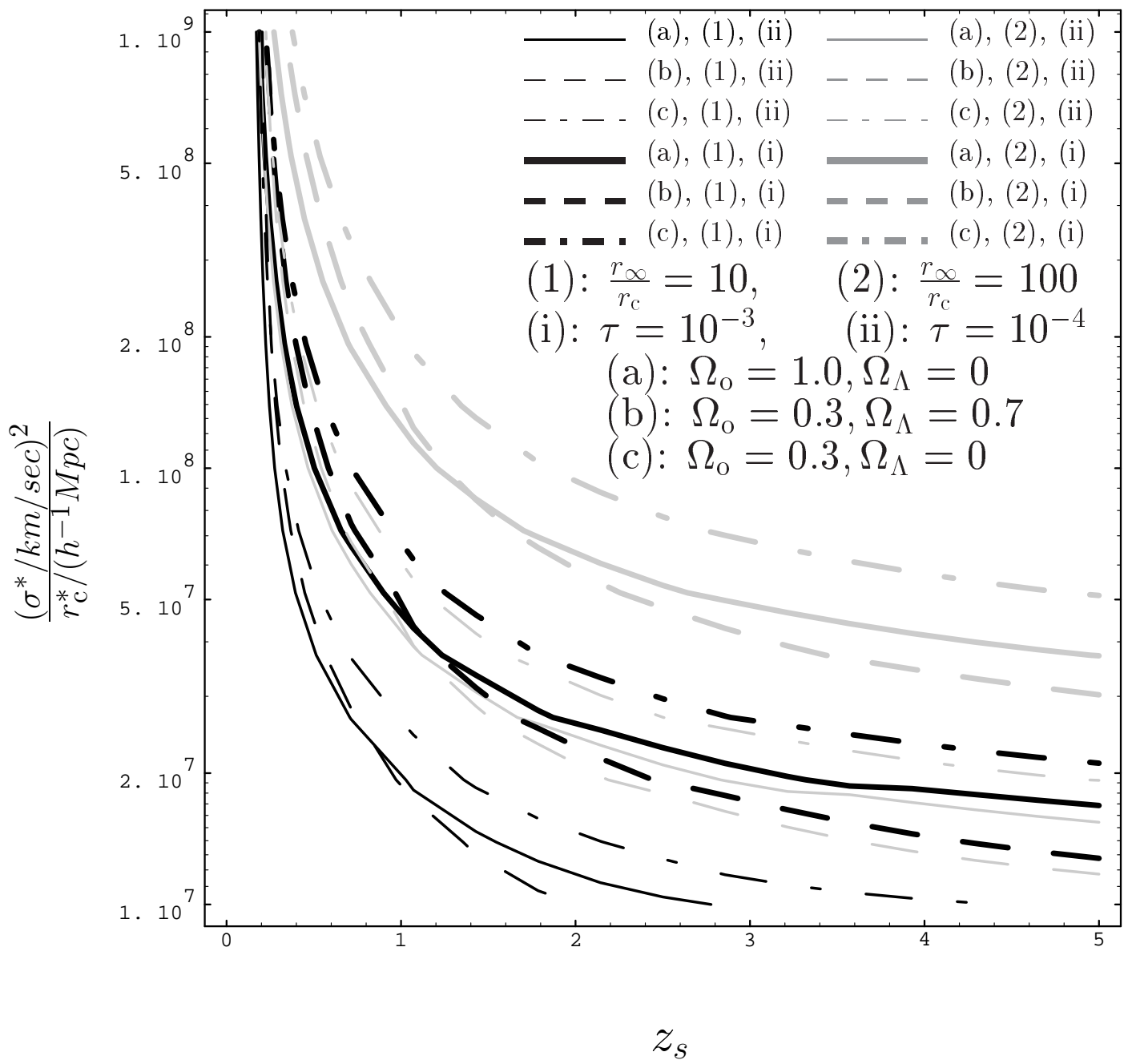

Fig. 3.- As for Fig. 2, but with $\alpha_{2}=-1.5$. Note that all curves involve smaller value of $\frac{\left(\sigma^{*} / k m / s e c\right)^{2}}{r_{c}^{*} /\left(h^{-1} M p c\right)}$ compared to those in Fig. 2. 


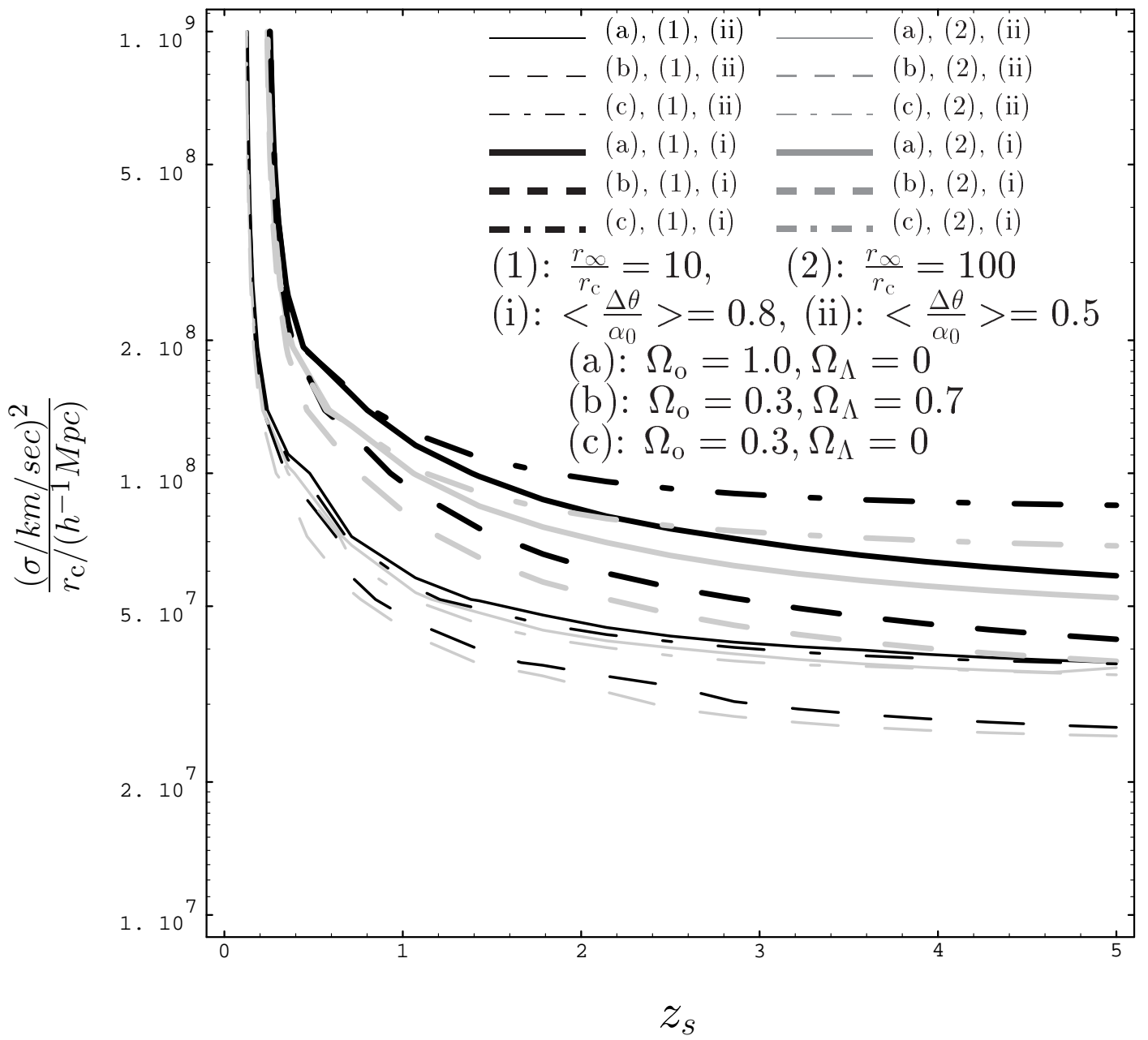

Fig. 4.- Contour plots of the expectation values of angular splitting normalized to that for an isothermal sphere, for fixed lens mass and showing contours $<\frac{\Delta \theta}{\alpha_{0}}>=0.5$ and 0.8 . The thicker width lines represent $\left\langle\frac{\Delta \theta}{\alpha_{0}}>=0.8\right.$, and the thinner width lines represent $<\frac{\Delta \theta}{\alpha_{0}}>=0.5$. All other features are as for Fig. 1 . 


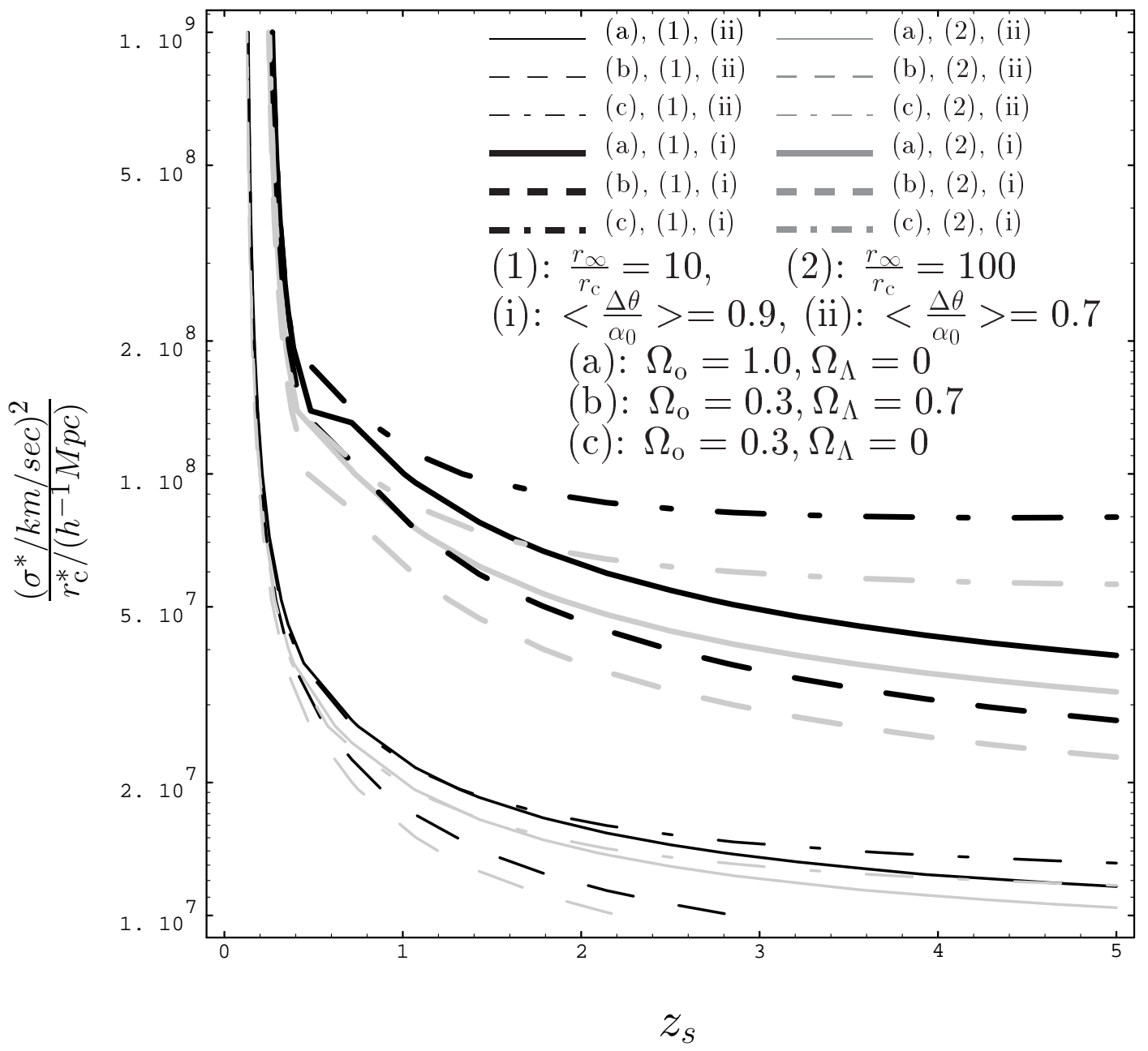

Fig. 5.- As for Fig. 4, but integrating over a Schechter distribution, with $\alpha_{2}=-0.5$ and showing, $<\frac{\Delta \theta}{\alpha_{0}}>=0.7$ and 0.9 . 


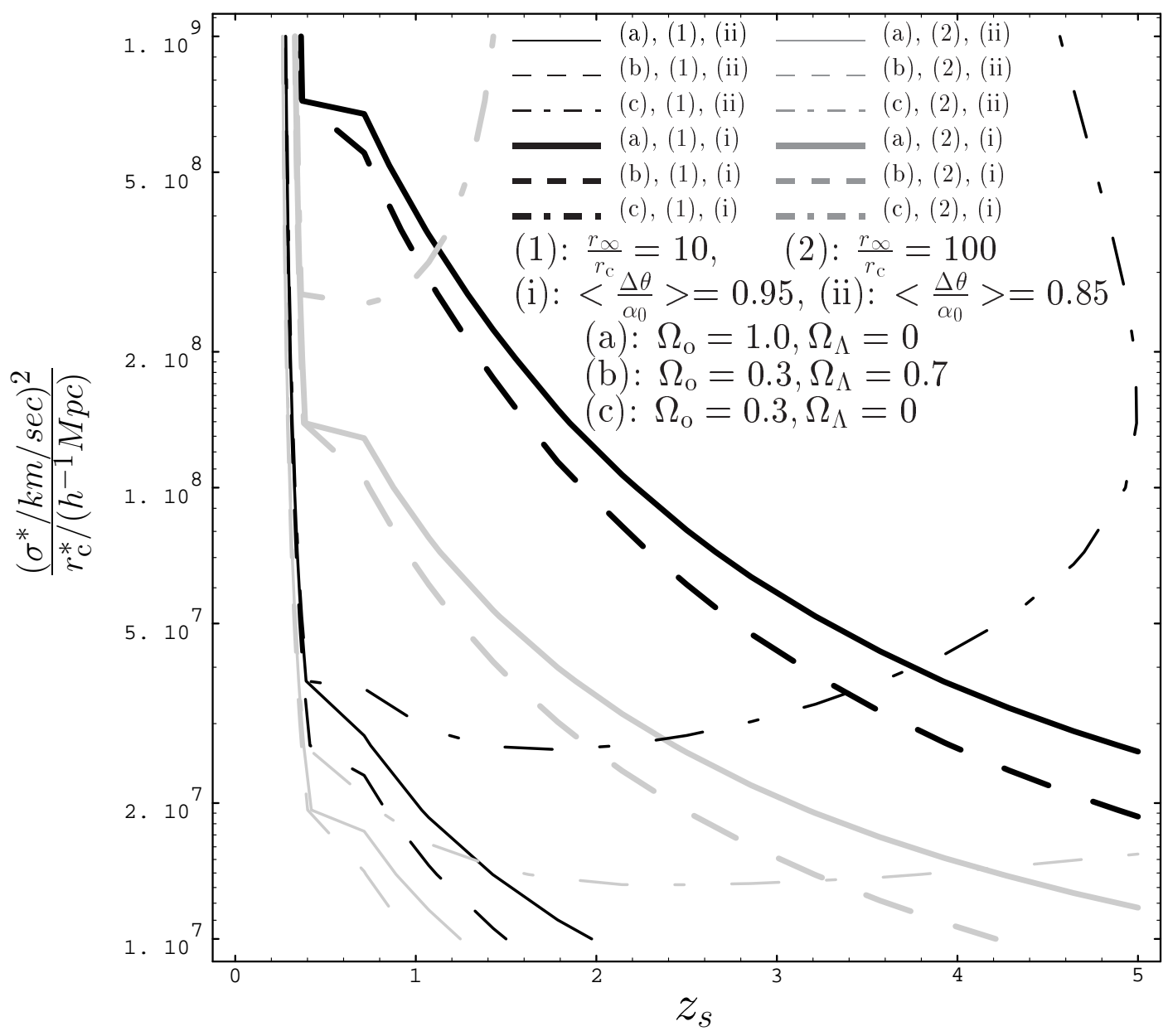

Fig. 6. - As for Fig. 5, but for $\alpha_{2}=-1.5$ and showing, $<\frac{\Delta \theta}{\alpha_{0}}>=0.85$ and 0.95 . Note that all curves involve smaller values of $\frac{\left(\sigma^{*} / \mathrm{km} / \mathrm{sec}\right)^{2}}{r_{\mathrm{c}}^{*} /\left(h^{-1} M p c\right)}$ compared to those in Fig. 5. 